\title{
La autonomía de las mujeres 'mayores' en el País Vasco y su contribución a los procesos de empoderamiento
}

\section{Marce Masa}

Adimen Investigación

<m.masa@adimen.es>

\section{Patxi Alija}

Adimen Investigación

\section{Estíbaliz Ibáñez}

Adimen Investigación

\section{Estíbaliz Berroeta}

Adimen Investigación

\section{Aurelio Alonso}

Adimen Investigación

Artikulu honetan aurkezten dira homonimoa den Emakunde-Emakumearen Euskal Erakundearen azterlanak xede harturiko hiru gai hauetako oinarrizko emaitzak: 65 urte eta gehiago dituzten emakumeen gaur egungo ahalduntze-prozesuen identifikatzea, erabakiak hartzean norberaren autonomia-mailaz izaten den pertzepzioa ezagutzea, eta egunerokotasunean kolektibo horrek izaten dituen gizarte-praktikak identifikatzea. Autonomia pertsonalaren maila altua duen emakume-belaunaldi bat izan arren, ez da oso zuzena ahalduntzearen kontzeptuak dituen dimentsio horiek guztiak aplikatzea. Aitzitik, nabarmentzen da emakume 'adindu' horien sozializaziorako eragile-rola, berauenean baino maila handiagoan, etorkizuneko beste belaunaldien ahalduntzean. Artikuluak defendatzen du, oro har, pertsona adinduen erabakitze-ahalmena bermatzea, eta partikularki emakume adinduen kasuan, zahartze aktiboaren izenean ekimenak abian jartzerakoan.

\section{HITZ-GAKOAK:}

emakumeak, pertsona adinduak, zahartzea, ahalduntzea, norberaren autonomia.
Este artículo presenta los resultados principales del estudio homónimo, impulsado por EmakundeInstituto Vasco de la Mujer, que persigue tres objetivos: identificar la situación actual de los procesos de empoderamiento de las mujeres de 65 y más años, conocer el grado de autonomía individual percibido en su toma de decisiones, e identificar las prácticas sociales cotidianas de este colectivo. Si bien se trata de una generación de mujeres con un elevado grado de autonomía personal, se considera poco ajustado aplicarles todas las dimensiones integradas en el concepto de empoderamiento. Por el contrario, se evidencia el papel de esta generación de mujeres 'mayores' como agentes de socialización del proceso de empoderamiento hacia otras generaciones de mujeres posteriores, más que en la suya propia. El artículo defiende la importancia de no perder de vista la autonomía de decisión individual de las personas mayores en general, y de las mujeres mayores en particular, cuando se ponen en marcha acciones en nombre del envejecimiento activo.

\section{Palabras clave:}

mujeres, mayores, envejecimiento, empoderamiento, autonomía individual. 


\section{Introducción}

En correspondencia a la relevancia adquirida por el empoderamiento en el IV y V Plan de Igualdad de Hombres y Mujeres del País Vasco elaborados por Emakunde (2006, 2010), esta entidad inició una línea de investigación para conocer la situación de este proceso en las prácticas cotidianas de las diversas generaciones de mujeres vascas. Este artículo presenta, concretamente, los resultados del estudio $L a$ autonomía de las mujeres 'mayores' en el País Vasco y su contribución a los procesos de empoderamiento (Emakunde, 2012), centrado en el colectivo de mujeres de 650 más años de edad ${ }^{1}$. Existe una investigación anterior orientada hacia el colectivo adolescente, chicas y chicos entre 14 y 17 años de edad (Emakunde, 2009), habiéndose publicado recientemente el estudio Los procesos de empoderamiento de las mujeres 'adultas' en el País Vasco, centrado en mujeres de 18 y 64 años de edad, que sirve de cierre a esta línea de investigación (Emakunde, 2013).

Se trata de una línea de investigación innovadora tanto en su temática como en su abordaje metodológico. Hasta el momento, las relaciones entre empoderamiento y mujeres han sido abordadas indirectamente, o no de forma sistemática, por escasos estudios, pues el empoderamiento constituye, como indicaremos a continuación, un concepto enmarcado más en el plano teórico que en la práctica empírica en las sociedades -denominadas-desarrolladas.

Y el carácter innovador del estudio en el caso concreto de las mujeres 'mayores' es aún mayor si cabe, porque todavía, incluso en el presente Año Europeo del Envejecimiento Activo y la Solidaridad entre las Generaciones (Comisión Europea, 2011) existe una cierta tendencia a 'cosificar' al colectivo de personas mayores, bien como contingente que implica retos demográficos y socioasistenciales de primera magnitud, bien como uno de los nichos de mercado más suculentos para las 'industrias de la salud' y las 'industrias del ocio y del consumo', sin pararse a preguntar, generalmente, cuál es la opinión concreta de las personas 'mayores' al respecto. Y esta búsqueda consciente de las opiniones de las personas mayores, en este caso, de las mujeres 'mayores', desde la perspectiva del empoderamiento, constituye el desencadenante de este estudio.

\section{Entender de manera contextualizada el concepto de empoderamiento}

Empoderamiento proviene de empowerment, ganar poder, fortalecerse. Como se ha reflejado en el IV y V Plan para la Igualdad de Mujeres y Hombres en la Comunidad Autónoma de Euskadi, el empoderamiento
${ }_{1}^{1}$ Queremos agradecer la absoluta disponibilidad brindada por Emakunde para la publicación de este artículo a partir del estudio indicado. y colectiva de las mujeres que les permite aumentar su participación en los procesos de toma de decisiones y de acceso al ejercicio de poder y a la capacidad de influir". (Emakunde, 2006: 44; 2010: 80).

El empoderamiento presenta un carácter integral, multidimensional, vinculado a la necesidad de potenciar la participación de las mujeres en todos los ámbitos de la vida social y, por ello, en la propia construcción de la identidad individual (Rowlands, 1997; Mosedale, 2003; Sánchez Álvarez, 2007; Fundación Isonomía, 2009). De ahí que se entienda el empoderamiento como un "proceso que no termina en lo individual, sino que trasciende al espacio colectivo" (Emakunde, 2010: 81).

El empoderamiento sería un proceso completado cuando se alcanzan los tres niveles, planos o subprocesos de los que se compone: el poder 'propio' (toma de conciencia personal o individual de las necesidades e intereses), vinculado íntimamente al concepto de autonomía; el poder 'con', que implica algún modo de organización social o colectiva para decidir sobre sus propias vidas; y, por último, el poder 'para', que implica una movilización política tendente a la transformación de las estructuras de subordinación y desigualdad de género (Murguialday y Vázquez, 2005: 43-44).

Sin embargo, como cualquier operativización empírica de un concepto, identificar la situación del empoderamiento y la capacidad de negociación en la toma de decisiones de las mujeres de $65 \mathrm{y}$ más años residentes hoy en el País Vasco requería, ineludiblemente, “traducir” (Latour, 1992: 117) la potencialidad y multidimensionalidad conceptual del empoderamiento a las características sociales e históricas de esta generación de mujeres, a sus propias realidades.

Se efectúa, por ello, una "perspectiva contextualizada" (León, 2001: 104) o "gradual” (del Valle et al., 2002: 49) del empoderamiento, que, en términos sociológicos más amplios, se apoyaría en las perspectivas “situadas" (Goffman, 1992) y “entrelazadas” (Simmel, 1986; y Elias, 1990), que remiten directamente a la dialéctica entre individuo y sociedad, entre agencia y estructura, sin duda, una de las raíces de la cuestión del empoderamiento y su grado de desarrollo.

El empoderamiento como proceso implica, por ejemplo, sostener que cualquier tipo de parámetro, indicador o índice de empoderamiento parte de las percepciones de personas situadas en contextos sociales, cursos de vida, pertenencias generacionales y de género determinados. Este planteamiento fue una de las principales conclusiones del Foro para la Igualdad de Emakunde 2009, pues el empoderamiento puede significar "cosas distintas para cada mujer, desde sus necesidades y aspiraciones, según su contexto personal, su momento vital y su entorno social" (Emakunde, 2009: 5). 


\section{La generación de mujeres 'mayores': contexto sociohistórico y de socialización}

Las mujeres nacidas antes de 1946 han vivenciado a lo largo de su trayectoria biográfica cambios difíciles de imaginar en buena parte de los planos de su existencia: condiciones materiales de su vida cotidiana, innovaciones tecnológicas, conquistas sociales y políticas, y un largo etcétera. Han vivido, posiblemente, en dos planetas diferentes, al menos.

Las mujeres de esta generación se hicieron jóvenes y adultas durante la dictadura franquista (Folguera, 1993; 1995; 1997). Este contexto, marco o estructura social y política adscribió un papel muy claro al género femenino: “ser-para-el hogar” (Murillo, 1996), "ser-para-los-otros" (Freixas, 2002: 261). Esta autonomía delimitada al escenario doméstico-familiar, a las prácticas de lo reproductivo, del cuidado; este centramiento en el escenario doméstico-familiar no evita que el estudio haya identificado que una de cada dos mujeres de 650 más años residentes en el País Vasco sí ha tenido alguna experiencia laboral a lo largo de su trayectoria biográfica.

Sin embargo, la ideología dominante en su proceso de socialización determinaba una segregación de género de los roles, las competencias y los escenarios sociales, donde lo público -lo laboral, lo político, lo asociativo u organizacional- constituía un ámbito masculino. Este substrato contextual sobre el que transitó esta generación de mujeres determina la imposibilidad de 'identificar' en ella un desarrollo en los planos social y político del empoderamiento, lo que obliga a focalizar el estudio en el plano individual o de autonomía del empoderamiento. En cierto modo, el concepto de empoderamiento no forma parte de su acervo de conocimiento (Schütz, 1992) en todas sus dimensiones.

Conscientes, como diría Weber (1964: 13), de que, en este caso, tal es el precio a pagar en cualquier opción metodológica, sostenemos que cualquier otra posibilidad constituiría forzar en demasía la búsqueda de las distintas dimensiones del empoderamiento en esta generación de mujeres 'mayores' cuando ello no es posible en términos sociológicos. Lo anterior no evita, como veremos más adelante, atender a las aportaciones de estas mujeres hacia la socialización de los distintos planos del empoderamiento no intrageneracionalmente, hacia sí mismas, sino intergeneracionalmente, hacia otras mujeres.

\section{La traducción empírica del empoderamiento: la elaboración del cuestionario}

Desde un punto de vista metodológico, en la línea indicada de atenuar, en lo posible, las dificultades de traducción del empoderamiento a las realidades de las mujeres ‘mayores' de 65 años residentes en el País Vasco, se optó por una combinación sucesiva de métodos cualitativos y cuantitativos. La aproximación cualitativa, concebida como estrategia de exploración, se plasmó a través de cuatro entrevistas en profundidad a personas 'informantes estratégicas' de la situación de esta generación de mujeres, y dos grupos de discusión, en los que han participaron directamente mujeres

El análisis de esta información cualitativa, junto a la bibliografía existente, permitió confeccionar un cuestionario ad hoc, pieza clave y, sin duda, uno de los mayores esfuerzos realizados en el estudio. Si el empoderamiento constituye un proceso de difícil traducción empírica o aplicada, sin duda porque se cuestiona a personas sobre aspectos que tienen que ver más con elementos intangibles, de opinión o subjetivos (Prieto et al., 2009) en este caso, su aplicación al colectivo de mujeres de 650 más años incrementó dicha complejidad, ante la necesidad de introducir una perspectiva panorámica o longitudinal por la trayectoria biográfica o curso de vista de esta generación de mujeres. Se requería, por otro lado, integrar en el cuestionario preguntas capaces de identificar de manera comparada la evolución -en aumento o declive- de la capacidad de autonomía y toma de decisiones por parte de estas mujeres.

Se efectuó un cuestionario amplio, compuesto por un total de 61 preguntas, con algo más de 500 posibilidades de respuesta, la mayor parte cerradas. El trabajo de campo se desarrolló entre la segunda mitad de octubre y primeros de noviembre de 2011, con una muestra de 600 mujeres en sus propios domicilios (límite de error estadístico de $\pm 5 \%$, para un nivel de confianza del $95,5 \%$ y $p=q=50$ ). Como se refleja en las Tablas 1 y 2, la muestra se distribuyó según el peso de cada colectivo en los tres territorios históricos y, dentro de éstos, según tres segmentos de población: municipios con más de 10.000 mujeres de esta cohorte de edad; con entre 2.000 y 9.999 mujeres; y, finalmente, con menos de 2.000 mujeres. La muestra, así como las rutas, con cinco cuestionarios por cada una de ellas, se distribuyó mediante una selección aleatoria, no efectuándose más de un cuestionario por portal. La elección de las rutas tomó como referencia la tipología de secciones censales del Instituto Vasco de Estadística-Eustat, que establece una diferenciación de las distintas secciones censales a partir de criterios socioeconómicos y socioculturales.

Tabla 1. Universo, por territorios históricos

\begin{tabular}{l|c|c|c|c}
\hline $\begin{array}{l}\text { Segmentos de } \\
\text { población }\end{array}$ & Álava & Bizkaia & Gipuzkoa & Total \\
\hline$>10.000$ mujeres & 21.152 & 57.840 & 22.515 & 101.507 \\
\hline $2.000-9.999$ mujeres & 0 & 37.424 & 20.570 & 57.994 \\
\hline$<2.000$ mujeres & 7.465 & 33.378 & 30.621 & 71.464 \\
\hline Total & $\mathbf{2 8 . 6 1 7}$ & $\mathbf{1 2 8 . 6 4 2}$ & $\mathbf{7 3 . 7 0 6}$ & $\mathbf{2 3 0 . 9 6 5}$ \\
\hline
\end{tabular}

Fuente: Elaboración propia a partir de la Estadística de Población y Viviendas del Eustat (EPVo6). 
Tabla 2. Distribución de la muestra, por territorios históricos

\begin{tabular}{l|c|c|c|c}
\hline \multirow{2}{*}{$\begin{array}{l}\text { Segmentos de } \\
\text { población }\end{array}$} & \multicolumn{4}{|c}{ Distribución de cuestionarios } \\
\cline { 2 - 5 } & Álava & Bizkaia & Gipuzkoa & Total \\
\hline$>10.000$ mujeres & 55 & 150 & 55 & 260 \\
\hline $2.000-9.999$ mujeres & 0 & 100 & 50 & 150 \\
\hline$<2.000$ mujeres & 20 & 90 & 80 & 190 \\
\hline Total & 75 & 340 & 185 & 600 \\
\hline
\end{tabular}

Fuente: Elaboración propia.

\section{La autonomía individual: el valor más preciado, hoy y mañana}

Es un hecho reiterado en los estudios aplicados orientados hacia el colectivo de personas mayores observar un alto nivel de satisfacción general respecto a sus condiciones de vida actual (Pérez Ortiz, 2003: 537; Sancho, 2010). En el caso de las mujeres de 65 y más años, ello también se ratifica, observándose 7,7 puntos de promedio de satisfacción general (Gráfico 1).

En el marco de la investigación, entre los elementos de satisfacción se incorporó uno que específicamente quería identificar la importancia, tanto en sí mismo, como en relación (o ranking) del factor 'capacidad para tomar decisiones (por sí misma)'. La autonomía individual en la toma de decisiones que afectan a la vida de esta generación de mujeres nacidas antes de 1946 y residentes en el País Vasco arroja unos niveles de satisfacción muy elevados. Además, se sitúa en segundo lugar, a la par en términos de satisfacción con las relaciones personales, por encima, incluso de los 'logros efectivos' que vienen alcanzado en su trayectoria vital.

Una de las conclusiones principales del estudio realizado por la Fundación Ingema tanto para mujeres como para hombres de 60 o más años de edad residentes en el País Vasco señalaba que "la autonomía empieza a ser considerada un valor social en alza en la vejez" (Sancho, 2010: 164). A la luz de nuestros resultados, y en el caso concreto de las mujeres de este grupo de edad, sostenemos que la autonomía individual es un valor social institucionalizado en esta generación de mujeres, un valor que, además, está guiando no sólo sus prácticas sociales actuales, sino también las próximas.

La denominada 'autonomía residencial' sería, sin duda, una de las manifestaciones patentes de esta traducción del valor de la autonomía individual en comportamientos actuales de las mujeres de 650 más años. La tendencia a permanecer en sus propios domicilios hasta edades muy prolongadas viene siendo indicada desde hace una década (Pérez Ortiz, 2003: 519), intensificándose y sedimentándose este 'envejecer en casa' en los últimos cinco años en el marco estatal (Pérez Ortiz, 2006a: 243), y reciente-

Gráfico 1. Niveles de satisfacción con distintos aspectos de su vida, por grupos de edad (escala 0-10)

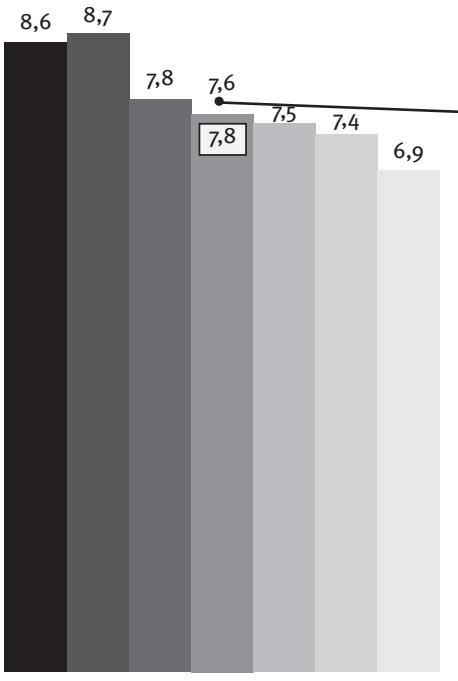

De 65 a 74 años

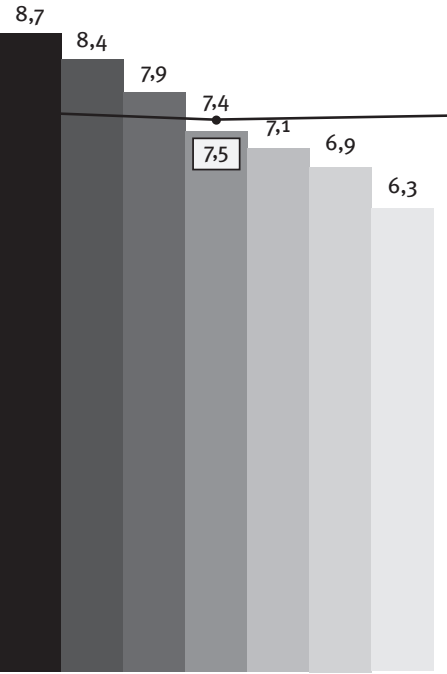

750 más años

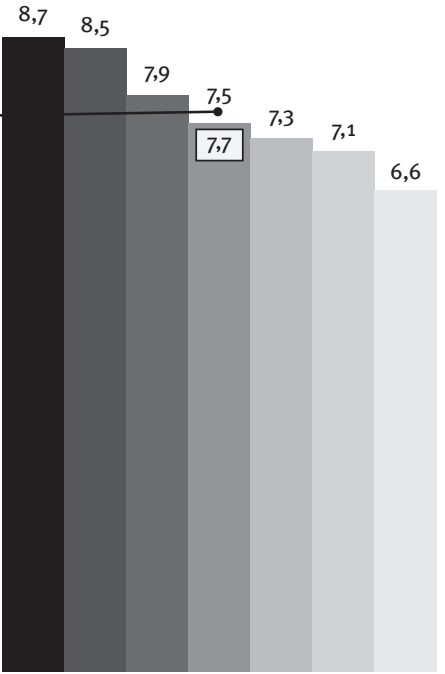

Total
Relaciones personales

Capacidad para tomar decisiones

Logros que está alcanzando en su vida

Nivel de vida
Sentimiento de pertenencia a una comunidad Estado de salud

Seguridad respecto a su futuro

Valores promedio

Fuente: Elaboración propia. 
mente en nuestro contexto autonómico y cotidiano más próximo (Sancho, 2010: 165).

Entre otros indicadores, la Tabla 3 muestra que las mujeres de 65 o más años residentes hoy en el País Vasco presentan un elevado grado de autonomía residencial. Además, su actual vivienda es su espacio de referencia, residen en ella una media de 36 años, y se mantienen igualmente en ella a lo largo de todo el año el 85,3\% de este colectivo de mujeres.

Tabla 3. Formas de convivencia, composición unidad familiar y tipo de residencia, por grupos de edad

\begin{tabular}{|c|c|c|c|}
\hline & $65-74$ años & 75 o más años & Total \\
\hline Sola & $21,1 \%$ & $39,9 \%$ & $31,0 \%$ \\
\hline En pareja & $47,9 \%$ & $30,7 \%$ & $38,8 \%$ \\
\hline $\begin{array}{l}\text { En pareja y con } \\
\text { hijos/as }\end{array}$ & $15,8 \%$ & $5,4 \%$ & $10,3 \%$ \\
\hline Con hijos/as & $7,0 \%$ & $10,8 \%$ & $9,0 \%$ \\
\hline Resto (otros modos) & $8,1 \%$ & $13,0 \%$ & $10,7 \%$ \\
\hline Total & $100,0 \%$ & $100,0 \%$ & $100,0 \%$ \\
\hline $\begin{array}{l}\text { Media de personas } \\
\text { en la misma vivienda }\end{array}$ & 2,1 & 1,9 & 2,0 \\
\hline $\begin{array}{l}\text { Edad media de la } \\
\text { pareja }\end{array}$ & 72,3 & 80,9 & 75,7 \\
\hline Mujeres con hijas/os & $90,5 \%$ & $86,4 \%$ & $88,3 \%$ \\
\hline Media hijas/os & 2,8 & 3,1 & 2,9 \\
\hline $\begin{array}{l}\text { Mujeres con nietas/ } \\
\text { os o biznietas/os }\end{array}$ & $75,4 \%$ & $78,5 \%$ & $77,0 \%$ \\
\hline $\begin{array}{l}\text { Media de nietas/os } \\
\text { o biznietas/os }\end{array}$ & 3,4 & 4,6 & 4,0 \\
\hline $\begin{array}{l}\text { Media de años que } \\
\text { vive sola }\end{array}$ & 12,3 & 13,4 & 13 \\
\hline $\begin{array}{l}\text { Media de años } \\
\text { residiendo en la } \\
\text { misma vivienda }\end{array}$ & 33,7 & 38,1 & 36,0 \\
\hline $\begin{array}{l}\text { Residencia todo el } \\
\text { año (\% afirmativo) }\end{array}$ & $83,5 \%$ & $87,0 \%$ & $85,3 \%$ \\
\hline
\end{tabular}

Fuente: Elaboración propia.

En este marco doméstico-residencial, e íntimamente vinculado a la necesidad de adaptar o contextualizar a cada colectivo (grupo, generación) el proceso de empoderamiento, el estudio ha puesto en evidencia el bajo nivel de empoderamiento económico formal de estas mujeres nacidas antes de 1946, y, por el contrario, el elevado nivel de empoderamiento en la gestión efectiva de la economía doméstica.

El primero de los planos no es más que el reflejo de las trayectorias de actividad principal hacia las que, de forma mayoritaria, se han orientado estas mujeres a lo largo de su curso de vida. De ahí que estas mujeres se encuentren, generalmente, con una jubilación determinada por la de su pareja masculina. Por este hecho, resulta ciertamente inapropiado demandar a estas mujeres 'mayores' la obtención de ingresos propios como requisito imprescindible para activar la dimensión económica del empoderamiento.
Tabla 4. La mujer como gestora de los gastos de la unidad de convivencia, por grupos de edad (\%)

\begin{tabular}{l|c|c|c}
\cline { 2 - 4 } & $65-74$ años & 75 o más años & Total \\
\hline Sí, básicamente yo sola & 69,4 & 73,4 & 71,5 \\
\hline $\begin{array}{l}\text { Sí, pero de manera } \\
\text { compartida }\end{array}$ & 26,4 & 16,5 & 21,2 \\
\hline No & 4,2 & 10,1 & 7,3 \\
\hline $\begin{array}{l}\text { Frecuencia: siempre ha } \\
\text { sido así, sola y compartida } \\
\text { (afirmativos) }\end{array}$ & 93,7 & 88,0 & 90,7 \\
\hline
\end{tabular}

\% afirmativos sólo en unidades de convivencia compuestas (dos o más)

\begin{tabular}{l|c|c|c}
\hline Sí, básicamente yo sola & 62,1 & 62,4 & 62,2 \\
\hline $\begin{array}{l}\text { Sí, pero de manera } \\
\text { compartida }\end{array}$ & 32,6 & 23,3 & 28,3 \\
\hline No & 5,4 & 14,3 & 9,4 \\
\hline $\begin{array}{l}\text { Frecuencia: siempre ha } \\
\text { sido así, sola y compartida } \\
\text { (afirmativos) }\end{array}$ & 94,6 & 87,8 & 91,5 \\
\hline
\end{tabular}

Fuente: Elaboración propia.

Por el contrario, más allá de la 'dependencia administrativa o legal' -de la fuente o procedencia de los ingresos económicos-, el bajo nivel de empoderamiento económico formal se transforma radicalmente si se fija la mirada en la práctica cotidiana, esto es, en la gestión de esos ingresos. Los resultados de la Tabla 4 no dejan lugar a dudas: esta generación de mujeres es la responsable de gestionar esos ingresos orientados a los gastos de la unidad de convivencia en más de nueve de cada diez casos (92,7\%). Y si atendemos sólo a las unidades de convivencia compuestas, es decir, neutralizando las mujeres de esta generación que reside en solitario, observamos que este rol también es desempeñado por el 90,5\%.

Otro aspecto muy destacable es la estabilidad a lo largo del tiempo de esta función de gestora económica de los gastos de la unidad de convivencia. Todo apunta a que este ámbito de decisión económica constituye una faceta en la que esta generación de mujeres trata de estar muy presente a lo largo de su vida, lo que incluye también su etapa actual. De hecho, los resultados de la investigación han constatado que este rol se delega, total o parcialmente, cuando la mujer requiere de ayuda en las tareas de la vida diaria, cuando su salud es percibida como mala o muy mala, o cuando reside con uno o varios de sus descendientes. Pero siempre, en todo caso, lo más tarde posible, pues delegar la capacidad de decidir sobre la gestión de los gastos de lo cotidiano denotaría la pérdida de la capacidad de tomar decisiones por sí misma, una merma de su autonomía individual, plasmada en su entorno vital más próximo.

En esta línea, y por identificar la traducción en prácticas o comportamientos futuros de este valor de la autonomía individual en las mujeres de 650 más años, el Gráfico 2 muestra el deterioro intelectual, el no valerse por sí mismas y, en menor medida, pero dentro de este mismo ámbito, la pérdida de la autonomía económica se convierten, sin duda, en la 
$9,1 \quad 9$,

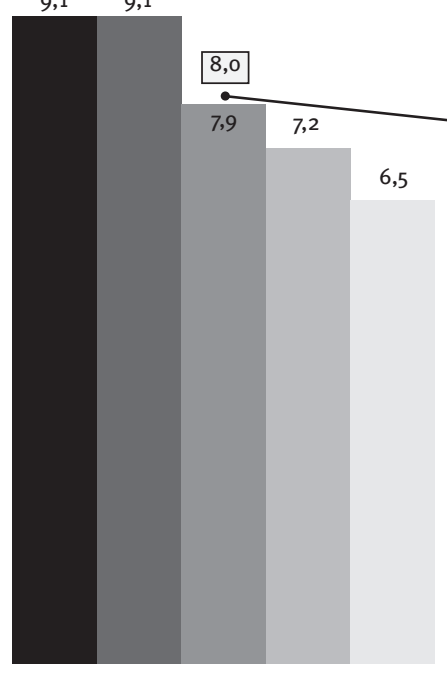

De 65 a 74 años
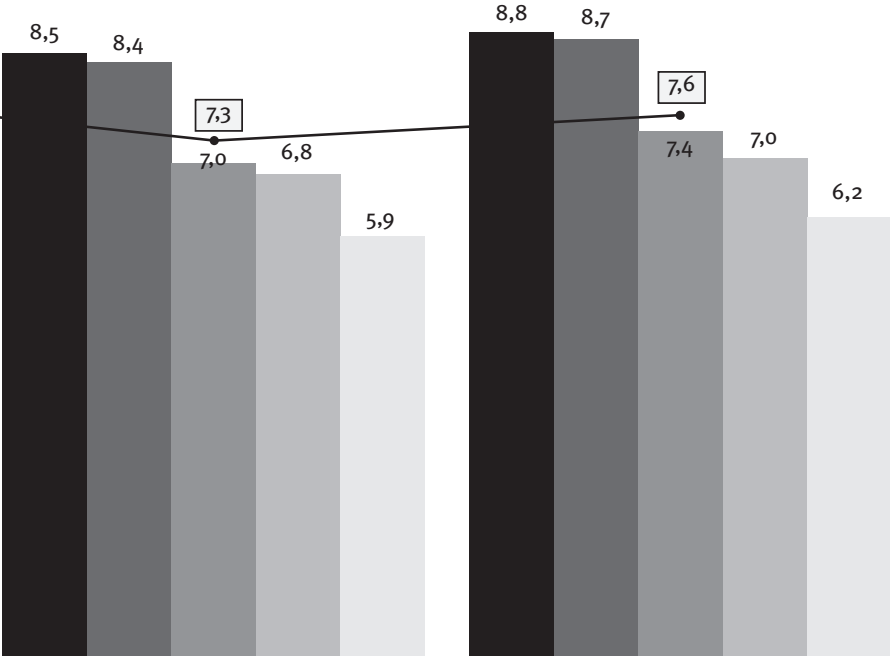

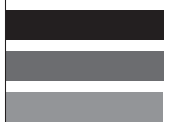

Deterioro intelectual

No valerse por sí misma

Pérdida de la independencia económica

5,9

Total

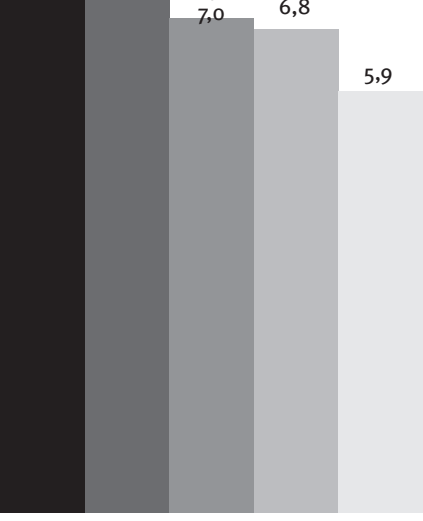

75 o más años

Deterioro físico

Pérdida de amistades (de vida social)

Valores promedio

Fuente: Elaboración propia.

preocupación más importante o vital para este colectivo de mujeres de la propia experiencia de envejecer.

En suma, la conquista de la autonomía individual como valor propio toma, por ello, un camino de no retorno dentro de esta generación de mujeres. Este valor presente y futuro no entiende, además, de diferencias según el nivel de estudios, la forma de convivencia, el nivel de ingresos mensuales y su procedencia, la trayectoria de la actividad principal desarrollada durante la vida adulta o el estado de salud percibida. Se trata, por lo tanto, de un valor transversal a esta generación de mujeres. Así, las mujeres de 650 más años serán "cada vez más libres de escoger su forma de vida en la vejez" (Pérez Ortiz; 2006a: 244).

\section{Distribución del tiempo cotidiano: prácticas sociales cotidianas domésticas, informales $y$ formales}

La Tabla 5 sintetiza de manera fotográfica cuáles son las prácticas sociales cotidianas de las mujeres nacidas antes de 1946, diferenciando tres grandes tipos: 1) aquellas que se desarrollan 'en el hogar' (home-centred); 2) aquellas que presentan un carácter 'social-informal'; y, por último, 3) aquellas que adquieren un carácter más 'formal u organizado'.

Ver la televisión, realizar las tareas de la casa y oír la radio son actividades muy presentes en esta generación de mujeres. Casi una de cada dos mujeres
(47\%) nunca desarrolla actividades relacionadas con el entorno doméstico (aficiones o hobbies), lo que refuerza el predomino de la televisión, la radio y, en menor medida, de la lectura como prácticas sociales cotidianas en las que transcurre el tiempo en este escenario doméstico-residencial.

El estudio no introdujo variables destinadas a conocer la capacidad de estas mujeres de decidir sobre los contenidos o temáticas de lo que ven en la televisión, escuchan en la radio o leen. Sin duda, ello podría constituir un tema de estudio en sí mismo, tan complejo como ilustrativo (Igartua y Badillo Matos, 2003), que esperemos pueda ser desarrollado con mayor profundidad en otros estudios. Seguramente atenuaría representaciones sociales que correlacionan, sin demasiadas evidencias, el denominado ‘ocio pasivo' y una colectividad abotargada o desentendida, de 'pan y circo', acaso con mayor énfasis en lo que se refiere a las personas mayores.

El espacio público ('la calle’), el otro gran escenario de un conjunto de prácticas cotidianas 'sociales informales'. Pese a la elevada presencia en él, hay importantes diferencias según actividades, a excepción de aquellas que se realizan en el entorno de residencia. El barrio o municipio, el entorno más próximo, es el lugar protagonista, donde se ponen en práctica el caminar, el acudir a parques públicos, el visitar a familiares, y las relaciones de amistad y de vecindad. Las cinco actividades sociales informales restantes arrojan, por el contrario, porcentajes comparati- 
Tabla 5. Actividades realizadas cotidianamente, según frecuencia, por grupos de edad (\%)

\begin{tabular}{|c|c|c|c|c|c|c|}
\hline \multirow{2}{*}{ Actividades } & \multicolumn{2}{|c|}{$65-74$ años } & \multicolumn{2}{|c|}{750 más años } & \multicolumn{2}{|c|}{ Total } \\
\hline & Sí & Nunca & Sí & Nunca & Sí & Nunca \\
\hline En el hogar (home-centred) & 100,0 & 0,0 & 100,0 & 0,0 & 100,0 & 0,0 \\
\hline Lee & 85,6 & 14,4 & 78,2 & 21,8 & 81,7 & 18,3 \\
\hline Oye la radio & 88,0 & 12,0 & 83,5 & 16,5 & 85,7 & 14,3 \\
\hline Ve la TV & 98,9 & 1,1 & 99,7 & 0,3 & 99,3 & 0,7 \\
\hline Tareas de la casa & 98,6 & 1,4 & 92,4 & 7,6 & 95,3 & 4,7 \\
\hline Actividades relacionadas con el entorno doméstico & 57,0 & 43,0 & 49,4 & 50,6 & 53,0 & 47,0 \\
\hline Sociales informales & 96,8 & 3,2 & 93,7 & 6,3 & 95,2 & 4,8 \\
\hline Actividades relacionadas con el entorno de residencia o barrio & 87,0 & 13,0 & 86,1 & 13,9 & 86,5 & 13,5 \\
\hline Actividades sociales (centro de personas ‘mayores’, salir) & 52,5 & 47,5 & 37,0 & 63,0 & 44,3 & 55,7 \\
\hline Acude a actos religiosos & 63,3 & 36,7 & 73,6 & 26,4 & 68,7 & 31,3 \\
\hline Actividades culturales (cine, teatro, conciertos) & 51,2 & 48,8 & 25,0 & 75,0 & 37,4 & 62,6 \\
\hline Usa nuevas tecnologías (Internet, correo electrónico) & 18,3 & 81,7 & 4,4 & 95,6 & 11,0 & 89,0 \\
\hline Hace turismo, viajes, excursiones & 62,5 & 37,5 & 28,3 & 71,7 & 44,5 & 55,5 \\
\hline Sociales formales u organizadas & 38,0 & 62,0 & 22,5 & 77,5 & 29,8 & 70,2 \\
\hline $\begin{array}{l}\text { Actividades físicas o deportivas (con sistematicidad en un } \\
\text { espacio) }\end{array}$ & 27,8 & 72,2 & 13,9 & 86,1 & 20,5 & 79,5 \\
\hline Actividades formativas (con sistematicidad en un espacio) & 15,2 & 84,8 & 7,9 & 92,1 & 11,4 & 88,6 \\
\hline Participa en organizaciones de voluntariado & 7,1 & 92,9 & 5,4 & 94,6 & 6,2 & 93,8 \\
\hline Participa en organizaciones vecinales & 2,8 & 97,2 & 1,9 & 98,1 & 2,3 & 97,7 \\
\hline Participa en organizaciones sindicales & 1,1 & 98,9 & 0,0 & 100,0 & 0,5 & 99,5 \\
\hline Participa en organizaciones políticas & 1,1 & 98,9 & 1,0 & 99,0 & 1,0 & 99,0 \\
\hline Participa en organizaciones de mujeres & 2,8 & 97,2 & 1,9 & 98,1 & 2,3 & 97,7 \\
\hline
\end{tabular}

Fuente: Elaboración propia.

vamente muy inferiores: algo más de una de cada dos mujeres de 65 o más años residentes en el País Vasco $(55,7 \%)$ nunca acude a un club de personas 'mayores' o centro de tercera edad, o nunca sale a comer o cenar con personas (amistades, familiares). Y el mismo porcentaje de ausencia de actividad se registra en turismo, viajes, excursiones (55,5\%). Las actividades culturales (cine, teatro, conciertos) nunca son realizadas por casi dos terceras partes de estas mujeres $(62,6 \%)$. Las actividades que requieren el uso de nuevas tecnologías (Internet, correo electrónico) son las que arrojan los indicadores más bajos: casi nueve de cada diez mujeres nunca las pone en práctica ( $89 \%$ ), lo que visualiza en toda su crudeza la brecha digital, que es generacional y de género, como se reflejó en el estudio de Castaño (2005).

Si atendemos, finalmente, al tercer nivel o tipología de prácticas sociales que adquieren un mayor grado de formalización u organización, la Tabla 5 muestra cómo dos de cada diez mujeres de esta generación $(20,5 \%)$ participan en actividades físicas o deportivas formalizadas (curso, club, polideportivo). Por el contrario, la presencia de esta generación de mujeres en organizaciones de participación 'institucional' (política, sindical, asociativa) es ciertamente residual. Sin duda, el tipo de socialización política que, como generación y como género, ha recibido este colectivo de mujeres es, de nuevo, clave para interpretar estos resultados. En todo caso, la participación en organizaciones de voluntariado parece que viene adquiriendo una acogida creciente entre esta generación de mujeres, el 6,2 \% del total, en especial entre aquellas que sienten que han ganado autonomía en esta etapa vital y las que tienen un nivel de estudios superior a la media. Se trata, sin duda, de una dimensión cuyo desarrollo habrá que seguir en próximos estudios.

\section{La continuidad identitaria como percepción generalizada}

Como decíamos, uno de los objetivos de este acercamiento a la situación del empoderamiento de las mujeres 'mayores' residentes en el País Vasco era comprobar hasta qué punto el cumplimiento de los '65 años', con tanto valor (hasta la fecha) desde el punto de vista simbólico y administrativo en nuestro entorno inmediato, suponía un cambio en la trayectoria biográfica de este colectivo de mujeres.

Como se refleja en el Gráfico 3, cumplir los 65 años supone, más bien, una continuidad en la trayectoria 
78,2

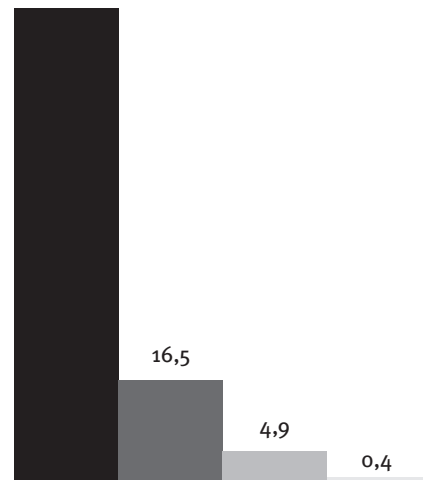

De 65 a 74 años
87,0

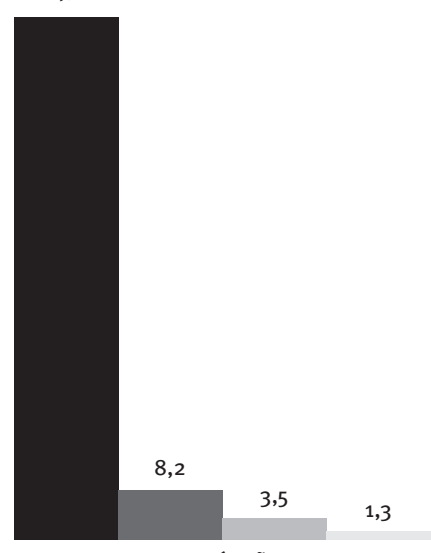

750 más años

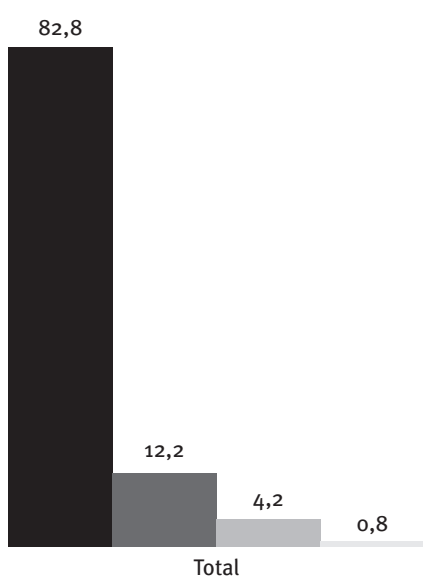

Una oportunidad para dedicar tiempo a otras cosas Otras respuestas

Fuente: Elaboración propia.

biográfica de esta generación de mujeres. Esta percepción de continuidad identitaria, de un sostenido y elevado nivel de autonomía individual en la gestión de las decisiones tomadas y que continúan tomándose, puede parecer, en sí misma, lógica, pues en nuestro contexto cultural cada persona efectúa una relectura ensamblada de su trayectoria biográfica, evitando, en lo posible, cualquier tipo de disonancia cognitiva.

Entre otras potenciales consideraciones, esta estabilidad en la estructura identitaria constituye un hecho de gran importancia en términos de la puesta en marcha de estrategias de actuación hacia las personas mayores. Si las identidades no se alteran de manera notable en la vejez (Pérez Ortiz, 2006b: 91), si “envejecemos como hemos vivido" (Freixas y Luque; 2009: 194), los márgenes para el cambio, para la activación de nuevas formas de pensamiento, de nuevas prácticas sociales, encuentran, cuando menos, un alcance o tope muy significativo, integrado en la propia estructura del carácter de cada persona mayor.

\section{Mayor disponibilidad de tiempo, que es liberado, no conquistado}

Una de las manifestaciones más palpables de esta continuidad en el tiempo de la forma de vida y de la identidad de las mujeres hoy ya 'mayores' tras haber traspasado la frontera de los 65 años se refleja en el Gráfico 4. La identificación de potenciales diferencias en la gestión del tiempo disponible en sus distintos planos de la vida cotidiana de esta generación de mujeres nacidas antes de 1946 residentes en el País Vasco permitiría conocer, igualmente, potenciales variaciones en su grado de empoderamiento indivi- dual actual. Sin embargo, se evidencia el predominio de la estabilidad en la orientación del tiempo en los aspectos analizados.

Y si bien la continuidad es relativamente homogénea -así lo reconocen, de media, seis de cada diez mujeres-, no lo es así en uno de los aspectos examinados: la disposición de un ‘tiempo propio', de un 'tiempo para sí misma', se ha incrementado significativamente $(40,3 \%$ en la categoría 'más ahora que antes'). No obstante, los resultados de la investigación corroboran que se trata de un tiempo liberado, que queda 'libre' por la desaparición de otros dos tiempos en liza, el tiempo de cuidado (por la emancipación de la descendencia) y el tiempo de trabajo productivo, presentes y dominantes en la anterior etapa adulta de su curso de vida.

Así, desde una perspectiva de empoderamiento, encontramos una generación de mujeres 'mayores' en la que su ganancia de tiempo no es, en términos generales, un tiempo conquistado, fruto de una estrategia (consciente) de empoderamiento individual, de toma de sus decisiones propias mediante la reorganización de las prioridades existentes, entre ellas, la de un tiempo propio, personal, destinado a cualquier actividad.

\section{Por un envejecimiento activo que no menoscabe la autonomía individual}

Prosiguiendo esta argumentación, resulta de gran interés reflexionar acerca de la capacidad de impulsar acciones y estrategias que doten de contenidos de empoderamiento a este tiempo (material) disponi- 


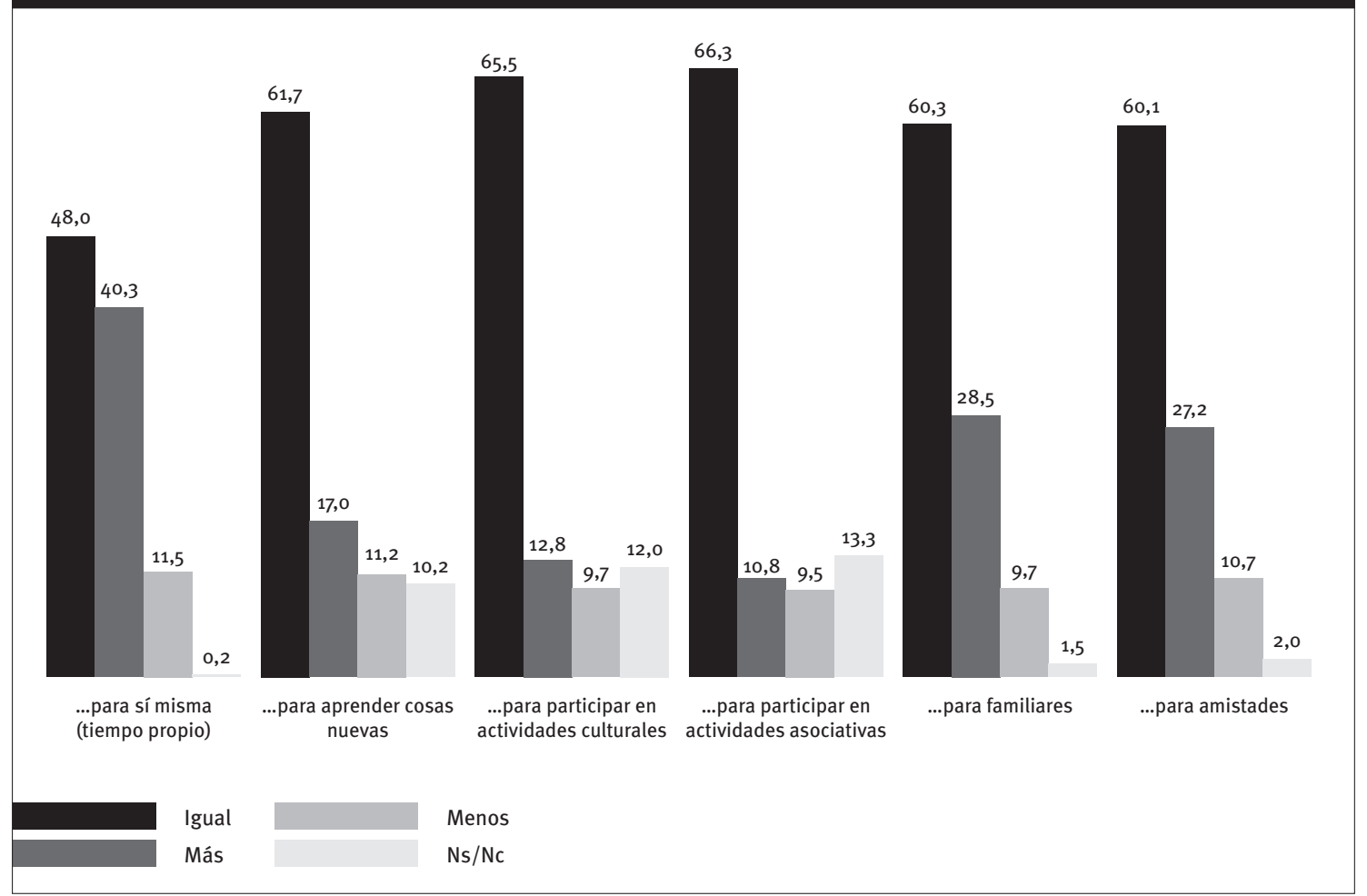

Fuente: Elaboración propia.

ble por parte de las mujeres de 650 más años. Aquí se situarían las distintas estrategias y programas del denominado 'envejecimiento activo y saludable', así como las 'industrias' del ocio, del consumo (Bódalo, 2003), de la salud o del cuidado que identifican a las personas mayores como uno de los nichos de mercado con mayor desarrollo.

Más allá de la autonomía individual que cada persona pueda tener a la hora de decidir la disposición de su tiempo como bien le parezca, en la generación de mujeres 'mayores' es evidente la influencia de factores estructurales -en este caso, culturales, de socialización- en el ritmo de desaparición de ese desajuste entre las potencialidades de tiempo disponible y las prácticas u orientaciones efectivas de esa misma disponibilidad de tiempo liberado. Nos parece muy acertada la interpretación de Pérez Ortiz cuando, a este respecto, indica que "muchos mayores [...] están todavía bastante alejados de la ética del derecho al tiempo libre y, por tanto, la ociosidad sigue siendo para ellos una conducta sancionable" (2006a: 246). Por esta razón, en la incorporación de las personas 'mayores' en general, y de las mujeres 'mayores' en particular, a las estrategias (acciones, programas, prácticas) del envejecimiento activo es necesario, todavía, un periodo de adaptación. Deberíamos, por ello, ser conscientes de la existencia de este 'ritmo', evitando estrategias que, por la presión de distintos grupos de interés, traten de forzar o acelerar su reducción, menoscabando la propia autonomía individual en la toma de decisiones de las personas.

\section{Envejecimientos 'en femenino': heterogeneidad de las mujeres 'mayores'}

La defensa de una perspectiva heterogénea, y no homogénea o unidimensional, del colectivo de personas mayores -que se plasma, paradigmáticamente, en la erradicación en el discurso de los términos 'vejez' o ‘viejos' frente al de 'mayores'-, viene siendo sostenida por distintos estudios desde al menos ya dos décadas (Fericgla, 1992; Sánchez Vera, 1993; Bazo; 1997), y recientemente en nuestro ámbito (Sancho, 2010: 163).

Además de aportar indicadores que refuerzan esta perspectiva multidimensional, el estudio La autonomía de las mujeres 'mayores' en el País Vasco y su contribución a los procesos de empoderamiento (Emakunde, 2012), al que estamos haciendo referencia en este artículo, identifica otro elemento de heterogeneidad en el colectivo de personas 'mayores', no ya relacionado con el género, sino 'intragénero'. Así, sería más apropiado hablar ya no tan sólo de un envejecimiento en femenino (Arber y Ginn, 1996; Freixas, 1997: 31; Pérez Ortiz, 2003; Rodríguez Rodríguez, 2010), sino de diversos envejecimientos en femenino según la edad. Esta idea ha sido reflejada con gran acierto por Pérez Ortiz (2006a: 13):

La vejez está cambiando de manera notable. La mejora del estado general de salud y la necesaria atención al problema de la dependencia invita a distinguir más que nunca dos vejeces, una 
autónoma y otra valetudinaria, o una tercera y cuarta edad. [...] En realidad, los cambios recientes indican que no hay una sola vejez, sino varias.

La diferenciación interna en dos grupos de edad, mujeres de entre 65 y 74 años de edad, por un lado, y mujeres de 750 más años, por el otro, ha permitido constatar la existencia de diferencias relevantes. Tan sólo a modo de ejemplo, se constata un mayor grado de actividad social por parte de las mujeres mayores más jóvenes (65-74 años), más activas en la puesta en práctica de actividades sociales informales. La edad continúa siendo un indicador útil para establecer parámetros de segmentación más específicos, si bien este tope de edad se marque cada vez en un tiempo más avanzado, y es seguro que ya se encuentra por encima de los 75 años.

Íntimamente ligada a la edad, se encuentra la salud, que juega, sin duda, un papel muy importante en esta percepción sobre cómo influye en cada persona la experiencia de envejecer, la estabilidad en su forma de vida adulta, especialmente, como obstáculo en la capacidad para tomar de manera autónoma las decisiones que le afectan en su vida. En todo caso, nuestro estudio identificó que seis de cada diez mujeres de 65 o más años residentes en el País Vasco consideran su estado de salud -percibido o subjetivo- como bueno o muy bueno.

\section{La socialización del empoderamiento: legado generacional y de género}

Donde no hay diferencias en esta generación de mujeres 'mayores' es en lo que denominamos la 'socialización intergeneracional del empoderamiento'. Venimos indicando que el concepto de empoderamiento resulta un término de escasa implantación en nuestras conversaciones diarias. También se ha señalado que el empoderamiento se plasma principalmente en el plano individual -como autonomía- en la generación de mujeres de 650 más años, por esas lógicas contextuales, sociohistóricas y de socialización que les han venido afectando. No obstante, si lo entendemos como un proceso de transformación social de las relaciones de género, como un proceso que trata de impulsar estrategias de acción para que las mujeres reconozcan y asuman sus propias capacidades para tomar las decisiones más oportunas en igualdad de condiciones, a este proceso de legado o trasmisión intergeneracional no ha sido ajena esta generación de 'mayores'.

Conscientes de la complejidad inherente a toda comparación intergeneracional (Mannheim, 1993), esta generación de mujeres nacidas antes de 1946 no ha sido mera espectadora del proceso de cambio operado en el progresivo incremento en la capacidad de autonomía de otras generaciones de mujeres en nuestro contexto. Diríamos que se trata de lo que podríamos categorizar como un empoderamiento proyectado, que se mantiene constante 0 en activo hasta la actualidad. Tal como refleja Tobío (2005: 86) en el caso concreto de las prácticas de cuidado de nietas y nietos, "las abuelas frecuentemente desean ayudar a sus hijas, en parte, porque a través de ellas pueden realizar una aspiración de independencia a la que no pueden acceder por sí mismas".

Esta socialización del empoderamiento, este legado generacional y de género, se plasma en dos hechos sociales institucionalizados. De una parte, la generación de mujeres 'mayores' presenta unos niveles de aceptación muy elevados respecto a la presencia de las mujeres, con independencia de su edad, en cualquier escenario de la vida social y sus prácticas correspondientes. Como hemos indicado en un apartado anterior, aunque la mayoría de las mujeres de 650 más años residentes en el País Vasco no participen de forma mayoritaria en actividades de ocio, culturales, formativas y asociativas, su ausencia en estos ámbitos no es impedimento para que acepten que otras mujeres, aún más si son de otras generaciones, tomen la decisión de hacerlo. El segundo hecho de esta socialización intergeneracional del empoderamiento hace referencia a que las mujeres 'mayores' creen que las mujeres pertenecientes a generaciones posteriores, es decir, sus hijas, sus sobrinas, sus nietas, son comparativamente más autónomas que ellas mismas. El Gráfico 5 no deja lugar a duda a este respecto en casi casi nueve de cada diez casos (87,2 \%).

La trasmisión intergeneracional de la importancia de que las mujeres tengan presencia en el escenario productivo-laboral es, sin duda, una de las formas más evidentes de esta estrategia de socialización intergeneracional del empoderamiento. "La idea central-como oportunamente ha señalado Tobío (2005: 83)- que tratan de trasmitirles a sus hijas es que lo principal, lo básico, es su propia autonomía individual concretada en su capacidad para mantenerse a sí mismas. [...] Lo más importante para una mujer es ahora esa autonomía básica individual centrada en el trabajo". Y este hecho adquiere un carácter $\tan$ transversal que, como se refleja en el Gráfico 6 , son incluso las mujeres de 650 más años que no han tenido presencia en el escenario productivolaboral las que recomiendan de manera más clara la presencia en dicho escenario, el tener un empleo. Se produce, por lo tanto, un aprendizaje vital, una experiencia no gratificante que tratará, en la medida de lo posible, de ser subsanada, de no ser reproducida, en el proceso de trasmisión generacional.

Las mujeres 'mayores' vienen siendo agentes activos del proceso de empoderamiento. Se trata de una agencia individual y generacional desplegada cotidiana y, quizá, en muchos casos, silenciosamente -por efectuarse en los escenarios doméstico-familiares, privados e incluso íntimos-, una socialización de la autonomía como valor central en la conformación de la identidad personal y género de las mujeres de generaciones posteriores. Desde la legitimidad que otorga la experiencia vivida, las mujeres nacidas antes de 1946 sostienen, en el fondo, que, pese a sus dificultades constantes y cotidianas, este legado ha tenido éxito en 


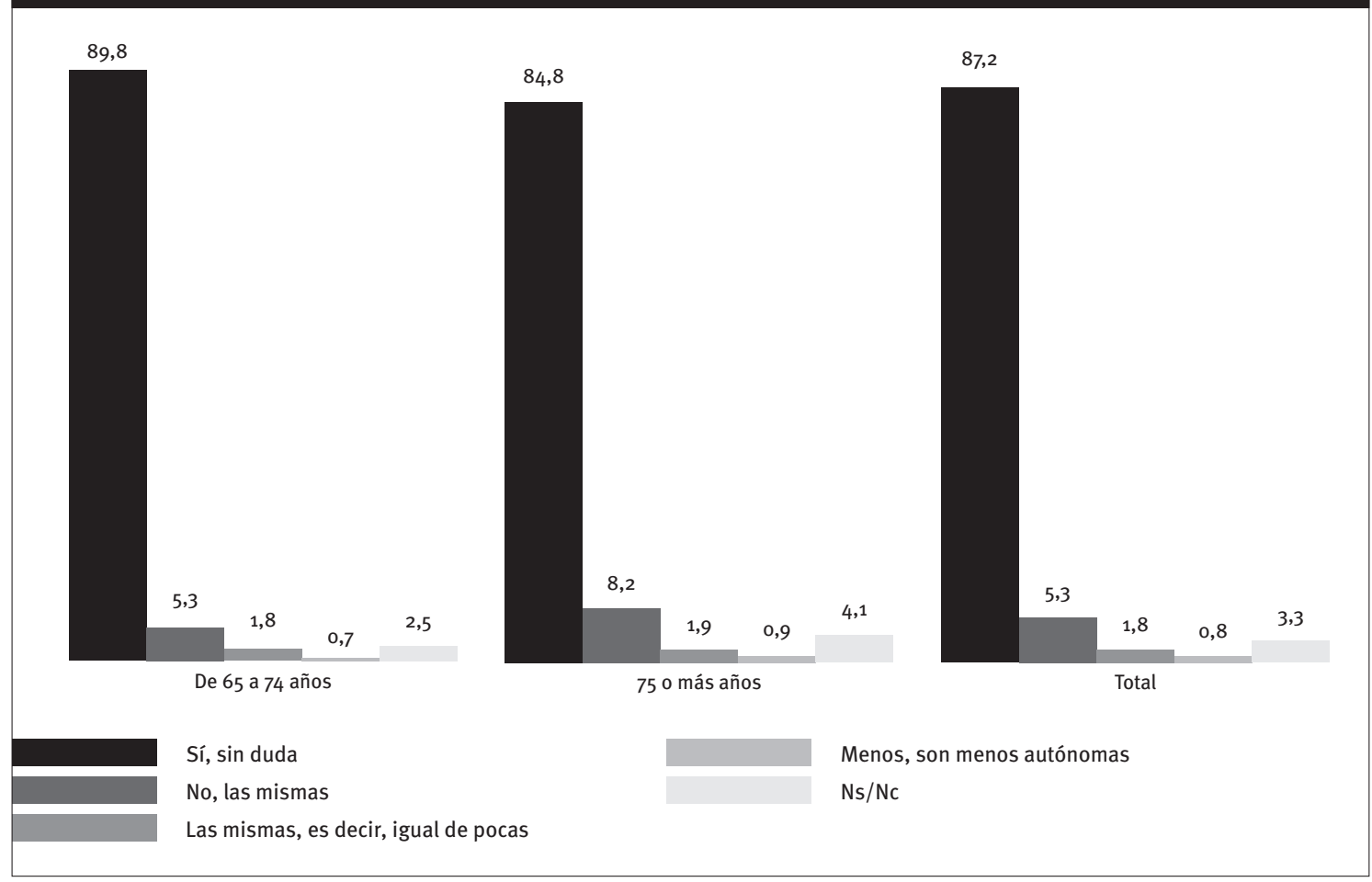

Fuente: Elaboración propia.

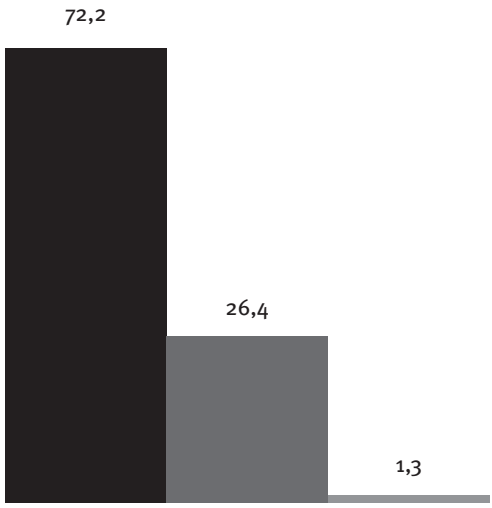

Presencia escenario laboral

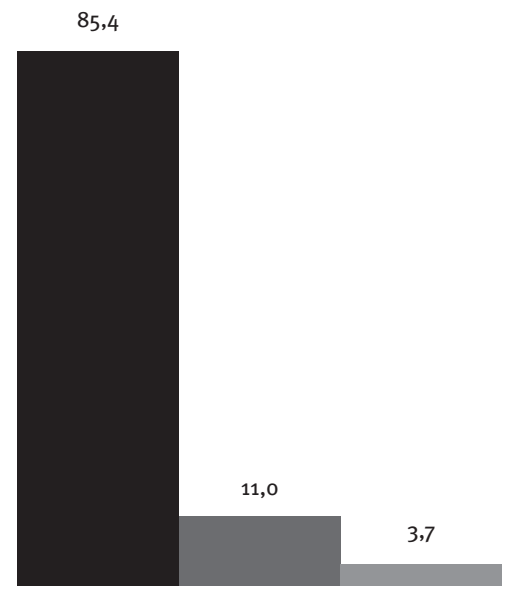

No presencia escenario laboral

\section{Sí recomendaría}

No recomendaría

$\mathrm{Ns} / \mathrm{Nc}$

Fuente: Elaboración propia.

términos de incremento en las condiciones de igualdad entre mujeres y hombres. Se trata de un proceso de cambio social estructural de tal magnitud e intensidad que no puede entenderse sin el apoyo, impulso y participación también de las mujeres 'mayores'. Como indica, de nuevo, muy acertadamente Lourdes Pérez Ortiz, "sin su experiencia previa y su contribución activa, nada de esto habría sido posible” (2006b: 90.) 


\section{Referencias bibliográficas}

ARBER, S.; y GINN, J. (1996): Relación entre género y envejecimiento. Enfoque sociológico, Madrid, Narcea.

BAZO, M. T. (1997): La sociedad anciana, Madrid, CIS.

BÓDALO, E. (2003): “Aproximación sociológica a las necesidades y al consumo de los mayores", Revista Española de Investigaciones Sociológicas, págs. 83-111.

CASTAÑO, C. (2005): Las mujeres y las tecnologías de la información. Internet y la trama de nuestra vida, Madrid, Alianza.

DEL VALLE, T. (coord.) (2002): Modelos emergentes en los sistemas y las relaciones de género, Madrid, Narcea.

ELIAS, N. (1990): La sociedad de los individuos, Barcelona, Gedisa.

EMAKUNDE (2013): La situación del proceso de empoderamiento de las mujeres 'adultas' en el País Vasco, Vitoria-Gasteiz, Emakunde.

- (2012): La autonomía de las mujeres 'mayores' en el País Vasco y su contribución a los procesos de empoderamiento, serie Informe, $\mathrm{n}$ 을 27 , VitoriaGasteiz, Emakunde.

- (2010): V Plan para la Igualdad de Mujeres y Hombres en la CAE. Directrices IX legislatura. VitoriaGasteiz, Emakunde.

- (2009): Adolescentes en Euskadi. Una aproximación desde el empoderamiento, serie Informe, $\mathrm{n}-23$, Vitoria-Gasteiz, Emakunde.

- (2009): VI. Berdintasunerako Gunea / Foro para la Igualdad 2009, Vitoria-Gasteiz, Emakunde.

- (2006): IV Plan para la Igualdad de Mujeres y Hombres en la CAPV. Directrices VIII legislatura, VitoriaGasteiz, Emakunde.
COMISIÓN EUROPEA (2011): How to Promote Active Ageing in Europe. EU Support to Local and Regional Actors, Bruselas, Comisión Europea [<http:// europa.eu/ey2012/BlobServlet?docld=7005\&l angld=en〉].

FERICGLA, J. M. (1992): Envejecer. Una antropología de la ancianidad, Barcelona. Anthropos.

FOLGUERA, P. (1997): “Las mujeres en la España contemporánea”, en GARRIDO, E. (ed.), Historia de las mujeres en España, Madrid, Síntesis.

- (1995): "La construcción de lo cotidiano durante los primeros años del franquismo", Revista Ayer, noำ19, págs.165-187.

- (1993): “Relaciones privadas y cambio social, 1940-1970", en FOLGUERA, P. (comp.), Otras visiones de España, Madrid, Editorial Pablo Iglesias.

FREIXAS, A. (2002): "Las mujeres queremos ser mayores y poder parecerlo", en MAQUIEIRA, $\mathrm{V}$. (comp.) Mujeres mayores en el siglo XXI: de la invisibilidad al protagonismo, Madrid, Ministerio de Trabajo y Asuntos Sociales [«http://www.imsersomayores.csic.es/ documentos/documentos/maquieiramujeres-01.pdf〉].

- (1997): “Envejecimiento y género: otras perspectivas necesarias", Anuario de Psicología, nํㅜㄱ, págs. 31-42.

FREIXAS, A.; y LUQUE, B. (2009): “El secreto mejor guardado: la sexualidad de las mujeres mayores, Política y Sociedad, vol. 46, no 1-2, págs. 191-203 [<http://revistas. ucm.es/index.php/POSO/article/view/ POSO0909130191A/21894)].

FUNDACIÓN ISONOMÍA (2009): Poder, poderes y empoderamiento... ¿Y el amor? ;Ah, el amor! 
Actas $5^{\circ}$ Congreso Estatal Isonomia sobre Igualdad entre mujeres y hombres, Castellón de la Plana, Universitat Jaume I [<http:// isonomiafundacion.uji.es/wp-content/ uploads/publicaciones/Actas_Congresos_ Estatales/V_Actas.pdf $>$ ].

GOFFMAN, E. (1992): “El orden de la interacción”, en GOFFMAN, E., Los momentos y sus hombres, Barcelona, Paidós.

IGARTUA, J. J.; y BADILLO, A. (coords.) (2003): Audiencias y medios de comunicación, Salamanca, Universidad de Salamanca.

LATOUR, B. (1992): Ciencia en acción, Barcelona, Labor.

LEÓN, M. (2001): “El empoderamiento de las mujeres: encuentro del primer y el tercer mundo en los estudios de género", La Ventana, no 13 , págs.94-106.

MANNHEIM, K. (1993): “El problema de las generaciones", Revista Española de Investigaciones Sociológicas, no 62, págs. 193-242.

MAQUIEIRA, V. (comp.) (2002) Mujeres mayores en el siglo XXI: de la invisibilidad al protagonismo, Madrid, Ministerio de Trabajo y Asuntos Sociales [khttp://www.imsersomayores.csic. es/documentos/documentos/maquieiramujeres-01.pdfs].

MOSEDALE, S. (2003): Towards a Framework for Assessing Empowerment, serie IARC Working Paper, no 03/2003, Manchester, Impact Assessment Research Centre [<http://purl.umn. edu/30578>].

MURGUIALDAY, C.; y VÁZQUEZ, N. (2005): Un paso más: una evaluación del impacto de género, ${ }^{\text {era }}$ edición, Barcelona, Cooperacció.

MURILLO, S. (1996): El mito de la vida privada. De la entrega al tiempo propio, Madrid, Siglo XXI.

PÉREZ ORTIZ, L. (2006a): La estructura social de la vejez en España. Nuevas y viejas formas de envejecer, serie Documentos Estadísticos, nํㅜ 20.010, Madrid, Imserso [<http://www.imserso.es/ InterPresent1/groups/imserso/documents/ binario/estructurasocialvejez.pdf`].
- (2006b): “Jubilación, género y envejecimiento”, en GIRÓ MIRANDA, J., Envejecimiento activo, envejecimiento en positivo, col. Biblioteca de Investigación, Logroño, Universidad de La Rioja [«http://dialnet.unirioja.es/descarga/ articulo/2756880.pdf)].

- (2003): Envejecer en femenino: las mujeres mayores en España a comienzos del siglo XXI, serie Estudios, no 81, Madrid, Instituto de la Mujer.

PRIETO, D. et al. (2009): Las dimensiones subjetivas del envejecimiento, colección Estudios, serie Personas Mayores, $\mathrm{n}$-11.007, Madrid, Imserso [shttp://www.imserso.es/ InterPresent1/groups/imserso/documents/ binario/11007dimensenvejec.pdf〉].

RODRÍGUEZ RODRÍGUEZ, P. (comp.) (2010): Mujeres. Las oportunidades de la edad, Madrid, Imserso.

ROWLANDS, J. (1997): Questioning Empowerment: Working with Women in Honduras, Oxford, Oxfam.

SÁNCHEZ ÁLVAREZ, P. (2007): Empoderamiento, recurso para conseguir la igualdad de géneros, Murcia, Ediciones Diego Martín.

SÁNCHEZ VERA, P. (1993): “Homogeneidad y diferenciación en la tercera edad. Bases para una sociología de la ancianidad", en SÁNCHEZ VERA, P. (ed.) Sociedad y población anciana, Murcia, Universidad de Murcia.

SANCHO, M. (dir.) (2010): Estudio sobre la realidad de las personas mayores de 60 años en el País Vasco. ECVPM-Euskadi, Vitoria-Gasteiz, Gobierno Vasco.

SCHÜTZ, A. (1992): El problema de la realidad social, Buenos Aires, Amorrortu.

SIMMEL, G. (1986): “El problema de la sociología”, en SIMMEL, G., Sociología 1. Estudio de las formas de socialización, Madrid, Alianza.

TOBío, C. (2005): Madres que trabajan: dilemas y estrategias, Madrid, Cátedra.

WEBER, M. (1964): Economía y sociedad, México D. F., Fondo de Cultura Económica. 